$63^{\text {ème }}$ Congrès de la SFCO, 02030 (2015)

DOI:10.1051/sfco/20156302030

(C) Owned by the authors, published by EDP Sciences, 2015

\title{
COMMUNICATION
}

\section{Lymphome folliculaire B : à propos de deux cas}

\author{
Montaner $\mathrm{S}^{*}$, Weingertner $\mathrm{N}^{* *}$, Marcellin $\mathrm{L}^{* *}$, Doustkam A-A*, Bornert $\mathrm{F}^{*}$ \\ * Unité de Chirurgie Buccale-Implantologie, Chirurgie Bucco-Dentaires, Hôpital Civil, CHRU de Strasbourg \\ ** Département de Pathologie, Hôpital de Hautepierre, CHRU de Strasbourg
}

Le lymphome folliculaire est une affection maligne du système lymphatique et un type courant de lymphome non hodgkinien. Le lymphome folliculaire provient des cellules $B$, et affecte principalement les personnes âgées et, en raison de sa croissance lente, est considéré comme un lymphome indolent (Schaaf et al. 2012).

Une manifestation primitive extra-nodale dans la cavité orale est extrêmement rare et se découvre chez des patients entre 60 et 70 ans sans prédilection de sexe.

Le cas d'une patiente de 81 ans traitée notamment pour une cardiopathie hypertensive et valvulaire, une insuffisance rénale chronique et une bronchite pulmonaire chronique obstructive est rapporté ici. Cette patiente est adressée par son chirurgien-dentiste traitant pour des avulsions dentaires et l'exérèse d'une lésion muqueuse. Lors de l'interrogatoire médical, la patiente ne décrit pas de signes d'altération de l'état général et l'examen clinique exobuccal ne montre pas d'adénopathies cervicofaciales palpables. Une hyperplasie palatine indolore recouvre le bord postérieur d'une prothèse à armature métallique. Cette lésion exophytiquenon ulcérée et à base sessile présente un tissu de recouvrement de couleur normal. Une biopsie de cette lésion de $1,5 \times 1 \mathrm{~cm}$ a conclu à un lymphome folliculaire de grade II selon la classification OMS 2008. Les investigations immunohistochimiques montrent que la prolifération de cellules lymphoïdes est positive avec les sérums dirigés contre CD 23, CD 20, CD10, bcl-2 et bcl-6. L’index de prolifération Ki67 est évalué à $30 \%$. Le contrôle local est satisfaisant. En raison de l'état général de la patiente, seul un traitement par injection hebdomadaire d'anticorps monoclonal rituximab a été entrepris.

Un deuxième cas concerne une patiente de 38 ans qui consulte pour un avis complémentaire à propos d'une tuméfaction chronique de la joue gauche siégeant dans le fond du vestibule. Elle avait bénéficiée d'une échographie et d'une IRM qui confirmait la présence d'une masse de consistance tissulaire évoquant un adénopathie chronique possiblement en rapport avec la dent 37 ou une tumeur d'une glande salivaire. L'examen clinique révèle la présence d'une masse ferme à la palpation mesurant approximativement $3 \mathrm{~cm}$ de long en mésio-distal. Une exploration chirurgicale sous anesthésie locale a permis de visualiser une masse d'aspect jaune clair située sous le plan du buccinateur. L'analyse anatomopathologique a mis en évidence un lymphome folliculaire B de grade III. Les anticorps dirigés contre CD20, bcl-6, CD10, CD23 sont positifs. Dans ce cas-là, il n’y avait aucun autre signe sur le plan général ou de douleurs décrites par la patiente.

This is an Open Access article distributed under the terms of the Creative Commons Attribution License 4.0, which permits unrestricted use, distribution, and reproduction in any medium, provided the original work is properly cited. 
$63^{\text {ème }}$ Congrès de la SFCO

Toute lésion atypique de la muqueuse et plus largement de la cavité buccale nécessite une analyse globale et une biopsie obligatoire pour écarter toute lésion maligne.

Nom et adresse du conférencier

Sylvain MONTANER

Unité de Chirurgie Buccale-Implantologie, Chirurgie Bucco-Dentaires, Hôpital Civil, CHRU de Strasbourg

1 place de l' hôpital

67091 Strasbourg (France)

montanersylvain@live.fr 